\title{
Continent ileocecal augmentation cystoplasty
}

\author{
Mark A Sutton ${ }^{1}$, John L Hinson ${ }^{2}$, Kevin G Nickell ${ }^{3}$ and Timothy B Boone ${ }^{4}$ \\ Scott Department of Urology, Baylor College of Medicine and the Veterans Affairs Spinal Cord Injury Unit, \\ Houston, Texas, USA
}

\begin{abstract}
Objectives: To evaluate the use of the ileocecal bowel segment for bladder augmentation in a select group of patients who need a low pressure, high capacity urinary storage mechanism and a continent, catheterizable, cutaneous stoma that, because of their physical limitations, is easier to catheterize than their native urethra. Methods: We reviewed records of 23 continent ileocecal augmentation cystoplasties performed over the last $5 \frac{1}{2}$ years. The goals of the operation, patient selection criteria, pre-operative evaluation, operative technique, and postoperative evaluation with results were studied. Results: Twenty-three patients underwent the procedure with the average follow-up being 26.9 months (range 3-67 months). Bladder capacity was increased by an average of 276.8 milliliters $(\mathrm{ml})$. No metabolic problems have been detected, and 95\% (22/23 patients) are continent via their urethra and stoma. Conclusions: This unique modification of the Indiana continent urinary reservoir is not technically difficult to create and is relatively free of complications. The bladder capacity is greatly increased and post-operative continence rates are excellent. Finally, the quality of life for these patients has been significantly improved by their ability to access the augmented bladder independently via an abdominal stoma.
\end{abstract}

Keywords: continent ileocecal augmentation; neuropathic bladder; cystoplasty

\section{Introduction}

Urinary incontinence can be a devastating problem for patients and their caretakers, especially when a coexisting neurological disorder limits the patient's ability to manage their urinary tract independently. Failure of pharmacological therapy and problems with urethral access to perform self-catheterization represent common dilemmas to the clinician trying to protect upper urinary tract function and restore continence. One possible option when this dilemma occurs is the continent urinary diversion.

The first continent urinary diversion is attributed to Simon in 1852 when he performed a ureterorectal anastomosis in a patient with a bladder extrophy. ${ }^{1}$ Since that initial pioneering effort there has been steady progress in the effort to create a reliable continent urinary diversion. Concomitantly, a gradual evolution has occurred in refining the optimal technique in bladder augmentation. Various segments of bowel, ureter, and even autoaugmentation in order

\footnotetext{
Correspondence: Timothy B Boone

${ }^{1}$ Resident, Scott Department of Urology, Baylor College of Medicine, Houston, Tx.

${ }^{2}$ Fellow, Neurourology and Urodynamics, Scott Department of Urology, Baylor College of Medicine, Houston, Tx USA.

${ }^{3}$ Fellow, Neurourology and Urodynamics, Scott Department of Urology, Baylor College of Medicine, Houston, Tx, USA.

${ }^{4}$ Assistant Professor, Scott Department of Urology, Baylor College of Medicine, Houston, Tx, USA
}

to create the ideal augmentation cystoplasty procedure have been used. The Indiana continent urinary reservoir and its subsequent modifications ${ }^{2}$ have enjoyed widespread success since the initial description of the reservoir in $1987 .{ }^{3}$ Sarsody described the technique of ileocecocystoplasty with a continent catheterizable stoma in 1992. ${ }^{4}$ His recent follow-up reported excellent results in eight patients followed for almost 3 years. ${ }^{5}$ We report similar results in 23 patients followed for a mean of 26.9 months. In the present study, we used a modification of the Indiana continent urinary reservoir technique in a distinct patient population. We review the patient characteristics and operative goals, describe the operative technique, and report our outcomes with this procedure.

\section{Materials and methods}

Between December 1990 and May 1996, 23 patients underwent continent ileocecal augmentation cystoplasty. The patient population included eight females and 15 males. Sixteen patients had spinal cord injuries and four had multiple sclerosis as the cause of the neuropathic bladders. One had 'non-neurogenic neurogenic' voiding dysfunction with loss of detrusor compliance and hydronephrosis. The remaining two patients had urethral loss, one secondary to urethral 
necrosis and the other due to a large bladder neck fistula that had not responded to several attempts at repair. The average age of the patients was 42.2 years (range 26-64 years).

The goal of the augmentation was to create a large capacity, low pressure, compliant reservoir $(>300 \mathrm{ml})$. It required a reliable continence mechanism both to the stoma and to an easily catheterizable cutaneous stoma that offered easier access than the native urethra. In our study, 19 of the 23 patients required the surgery both to augment their bladder capacity and to create a continent, catheterizable, cutaneous stoma (Table 1, Group 1). The remaining four patients required the surgery mainly for the continent stoma needed particularly because of urethral trauma or because of their inability to catheterize their native urethras (Table 1, Group 2).

Among the selection criteria were that the patients have a life expectancy of more than 1 year, have manual dexterity sufficient to perform the catheterization of the abdominal stoma, have a strong motivation to be 'bag free', have adequate renal and bowel function, and have physical/social limitations that made catheterization via the native urethra difficult and/or impossible. If high grade vesicoureteral reflux was present, then ureteral reimplantation was performed in the same setting. Anti-incontinence procedures to the urethra were performed concomitantly in 11 of the 23 patients: seven urethral closures, three pubovaginal sling procedures, and one collagen injection. Finally, one patient underwent a concurrent right nephrectomy secondary to non-function of that kidney.

Preoperative evaluation included a detailed history and physical examination designed to assess the patients' goals and level of function, routine blood chemistry evaluation, an upper tract study, a barium enema in patients with a history of gastrointestinal disease, and a flourourodynamics study. The decision regarding either urethral closure or functional bladder neck closure (pubovaginal sling) was based on both history and flourourodynamic findings. Patients with neuropathic bladders who complained of preoperative incontinence and those in whom an open bladder neck and incontinence were demonstrated on video studies underwent either urethral closure or pubovaginal sling, depending on gender and surgeon's preference. The enterostomal therapy service and occupational therapy service counseled patients on self-catheterization and marked their stomal site pre-operatively. All patients had to demonstrate the manual dexterity necessary to perform the self-catheterization technique. All patients had a mechanical bowel preparation.

The operative technique is a modification of the Indiana continent urinary reservoir procedure. The ileocecal segment is isolated and the bowel reanastomosed. The efferent limb is tapered over a 12 French (F) catheter using a GIA-60 stapling device, and the area of the ileocecal valve is reinforced, as previously described. $^{3}$ The large bowel is then opened along its tenia in an anti-mesenteric fashion in preparation for its anastomosis with the bladder. The urethral closure or pubovaginal sling usually is completed prior to isolation of the bowel segment. Urethral closure in the female is completed from above and below to assure a multi-layer closure. The sling is fashioned from rectus fascia, suspended on prolene sutures, placed at the bladder neck, and utilized for functional closure of the urethra.

The bladder is then opened transversely along its anterior surface, and the detubularized right colon is sewn to the opened bladder (Figure 1). A suprapubic tube is placed through the native bladder prior to complete closure. The augmentation is then filled to check for leaks and pressure is applied to the augmentation with the drainage tubes clamped to assess continence of the efferent limb. A $14 \mathrm{~F}$ stomal catheter is placed to drain the bladder and ensure easy passage through the efferent limb into the newlyaugmented bladder. A flush right lower quadrant stoma is created according to Rowland's previously described technique. ${ }^{3}$ Finally, a closed suction drain is placed in the pelvis. We routinely use gastrostomy tubes in this patient population because of concomitant neurogenic bowel dysfunction.

Postoperatively, irrigation of the pouch is begun on the first post-operative day to prevent mucous buildup. A pouch-o-gram is performed after a week to 10 days, and the drain is removed if no leak is demonstrated and the drain output is less than $50 \mathrm{ml} /$ day. Assuming adequate return of bowel function, the patient is discharged with the suprapubic tube and plugged stomal catheter in place and the gastrostomy tube, clamped, and in place. Irrigation through the suprapubic tube is done at home approximately four times daily. At 3 weeks postoperatively, the patient returns to the office, where the stomal catheter is removed and the suprapubic tube is clamped. Selfcatheterization is then taught, with the catheterization time schedule at first being every 2 to $3 \mathrm{~h}$ and gradually increasing to every 4 to $6 \mathrm{~h}$. The suprapubic tube is removed after the patient is able to catheterize the stoma independently for 1 week.

\section{Case example}

$\mathrm{SC}$ is a 48-year-old female with a history of multiple sclerosis (MS) for 21 years. Over the past several years she has developed debilitating urgency and urge incontinence (voiding small volumes every $1-2 \mathrm{~h}$ and using $8-10$ pads/day). These effects were seen despite high doses of both Probanthine and Ditropan. Otherwise, she was healthy, but her lower extremity spasticity from MS made catheterization of her native urethra extremely difficult. Her creatinine was 0.8 ; she had no history of urinary tract infections, and bowel function and upper tract studies were normal. Urodynamics revealed consistent urinary incontinence secondary to detrusor hyperreflexia with a low capacity bladder (122 ml) (Figure 2). On the fluoroscopic 


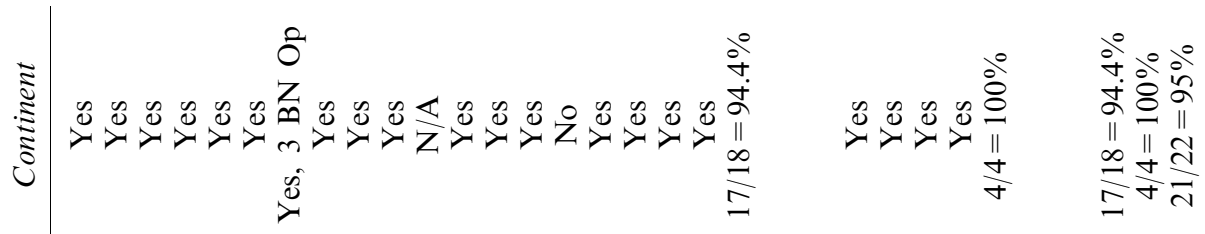

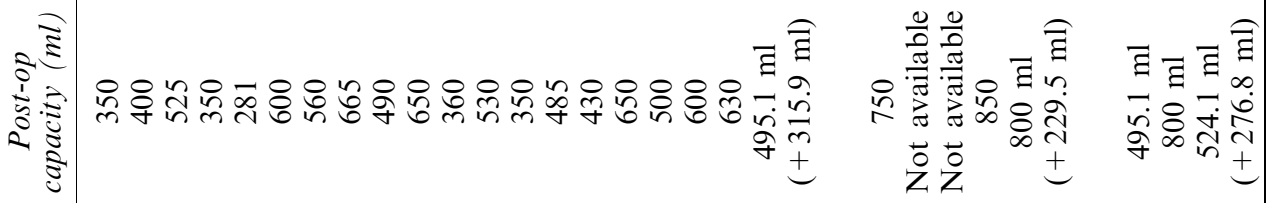

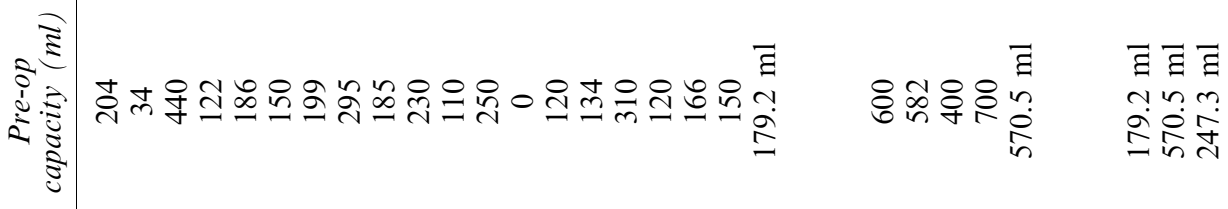

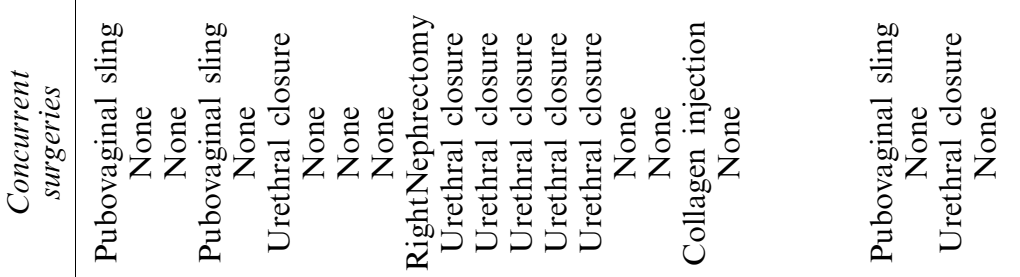

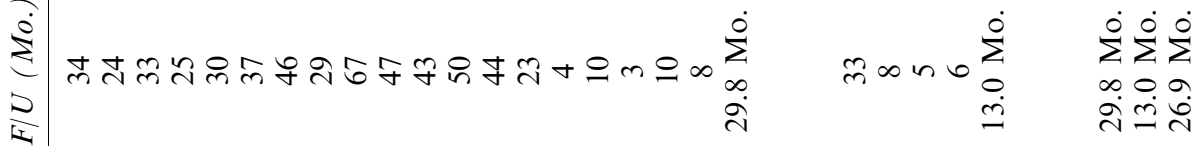

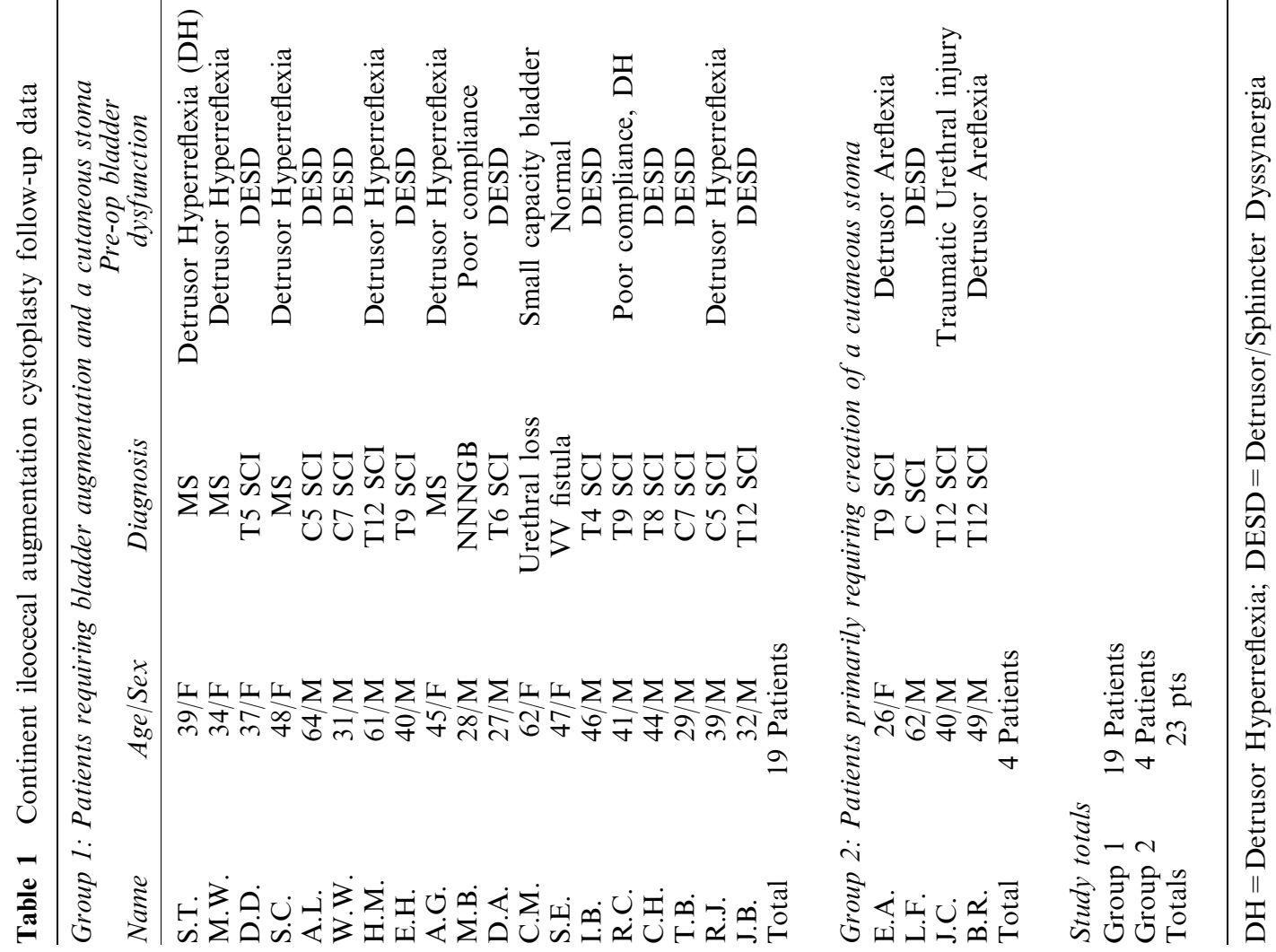




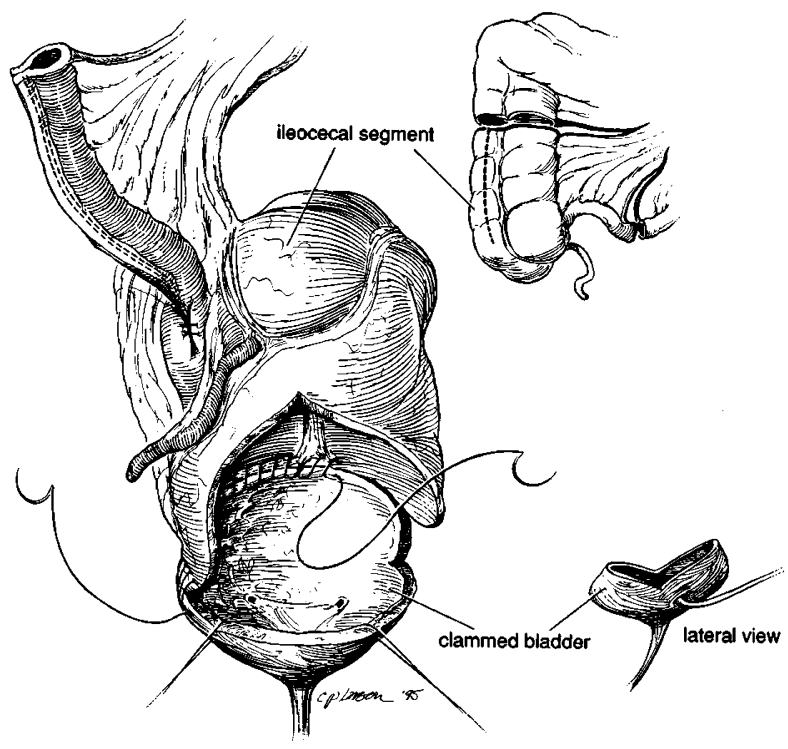

Figure 1 The continent ileocecal augmentation cystoplasty with diagram of the segment of bowel taken in the upper right, and the view of the frontal plane cystotomy in the lower right

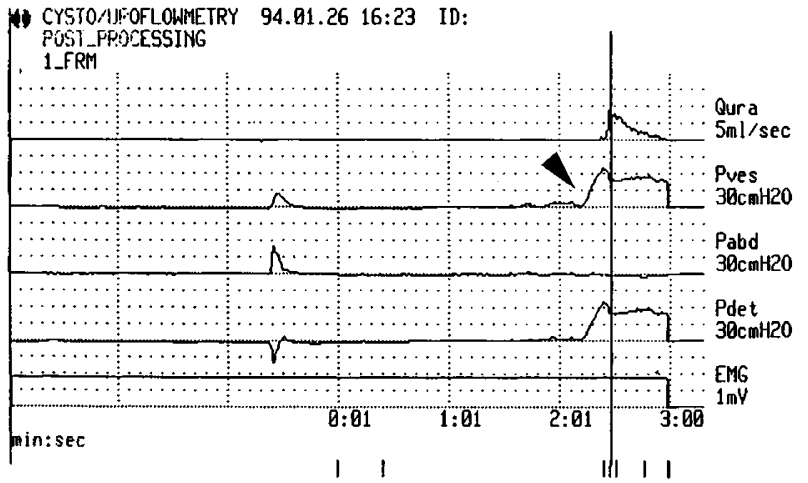

Figure 2 Pre-operative urodynamic findings in case report patient demonstrating a low capacity bladder of $122 \mathrm{ml}$ and detrusor hyperreflexia. Note the volume at capacity was $122 \mathrm{mls}$ (see arrow)

portion of the study, her bladder neck was found to be hypermobile with early opening causing leakage of urine.

The patient underwent a continent ileocecal augmentation cystoplasty and a pubovaginal sling procedure. Her bladder capacity was increased from $122 \mathrm{ml}$ to $350 \mathrm{ml}$ and her urinary symptoms were completely eliminated. Post-operative urodynamics revealed a compliant, low pressure reservoir with a capacity of $350 \mathrm{ml}$ (Figure 3). She is able to catheterize her abdominal stoma independently and is continent from her native urethra and newly formed stoma.

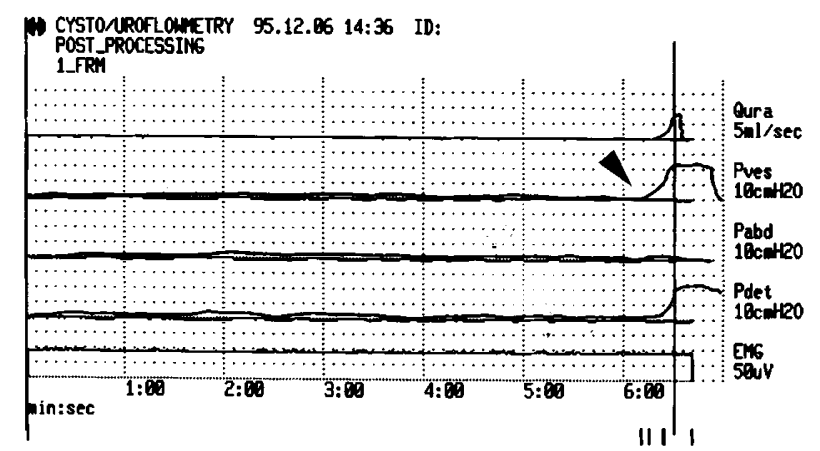

Figure 3 Post-operative urodynamic findings in case report patient demonstrating a compliant augmented bladder with a capacity of $350 \mathrm{ml}$. Note the volume at capacity was $350 \mathrm{mls}$ (see arrow)

\section{Results}

Twenty-three patients underwent continent ileocecal augmentation cystoplasty with the operative outcomes described in Table 1. These patients were treated over a period of 51/2 years, from December 1990 to May 1996. Mean follow-up was 26.9 months (range 3-67 months). All study patients were evaluated urodynamically in follow-up in order to determine and document post-operative augmentation capacity and continence. One patient was lost to follow-up before his post-operative evaluation and one patient has not yet undergone post-operative urodynamic testing.

Patients were divided into two groups. Group 1 patients $(n=19)$ required an augmentation cystoplasty and a continent catheterizable stoma. Therefore, the operative indication for these patients was both an increase in capacity and a continent pathway to access the augmented bladder. Group 2 patients $(n=4)$ were unable to catheterize via the urethra and needed a new route to drain their bladder. Of all patients, the average increase in bladder capacity was $276.8 \mathrm{ml}$ (range $85-480 \mathrm{ml}$ ). The overall average post-operative capacity was $524.1 \mathrm{ml}$ (range $281-850 \mathrm{ml}$ ). In group 1 the average follow-up was 29.8 months (range 3-67 months), and bladder capacity was increased by $315.9 \mathrm{ml}$ (from an average of $179.2 \mathrm{ml}$ to $495.1 \mathrm{ml}$ ). Group 2 patients had an average follow-up of 13.0 months (range 5-33 months), and their bladder capacity was increased by $229.5 \mathrm{ml}$ (from 570.5 to $800 \mathrm{ml}$ ).

Additional explanation is necessary to understand the procedure as performed in the group 2 individuals. These patients already had acceptable bladder capacities, but were unable to easily access their bladders because of physical limitations. For example, lower extremity spasticity can prevent self catheterization because the urethral meatus is not easily accessible (as in patients EA and BR in the table). Minimal augmentation was performed in these individuals. 
The surgical objective for these patients was mainly to create a continent, catheterizable stoma with the available anatomy. The appendix, which is often used as a catheterizable channel, was missing in these individuals and necessitated the use of the ileocecal segment. If the bladder outlet resistance measured preoperatively was adequate to maintain continence, then no urethral procedure was required to increase the outlet resistance (group 2, patient BR). However, patients such as EA (group 2) required additional surgery (pubovaginal sling) to increase the outlet resistance at the native urethra to maintain continence.

The surgical time required to perform this procedure is approximately $4 \mathrm{~h}$ and no blood transfusions were required. Hospitalization time averaged 7 days and mainly depended upon return of bowel function. No further surgery was required on the bowel and no surgical complications were detected. At some time during their follow-up period, four of the 23 patients developed febrile urinary tract infections that were easily treated with oral antibiotics. Only two of the 23 patients required re-operation. One patient required three urethral closure procedures to establish urethral continence and another required repeat collagen injection in the bladder neck to establish urethral continence. With close follow-up, none of the patients have thus far developed metabolic problems. Finally, 22 of 23 patients $(95 \%)$ maintain continence by cutaneous stoma and by urethra.

\section{Discussion}

Patients who traditionally have had their urinary systems managed with either an indwelling urinary catheter, sphincterotomy, or with an incontinent diversion (with or without a cystectomy) are candidates for this procedure. The surgical technique described has several distinct advantages over traditional techniques. First of all, no indwelling foreign body is present that potentially can lead to urinary tract infections, urinary calculi, or cancer formation. Second, maintaining the bladder and trigone avoids the potential for pyocystis in the retained bladder after incontinent urinary diversion. It allows the augmentation segment to be anastomosed directly onto the native bladder in a manner similar to the technique of Bramble $^{16}$ and Mitchell and Rink, ${ }^{7}$ thus shortening the surgical time and avoiding the potential adverse sequelae of new ureteral anastomosis. If significant ureteral reflux is detected during the pre-operative evaluation, then ureteral reimplantation is easily performed into the native bladder, and not into the less reliable augmented bowel segment. The bladder capacity is increased by an average of $276.8 \mathrm{ml}$, thus greatly increasing the time interval between urethral catheterizations. Finally, this technique maintains fertility potential and the possibility of collecting semen for use in assisted reproduction.

Most of our study patients had either spinal cord injuries or multiple sclerosis. These patients require a

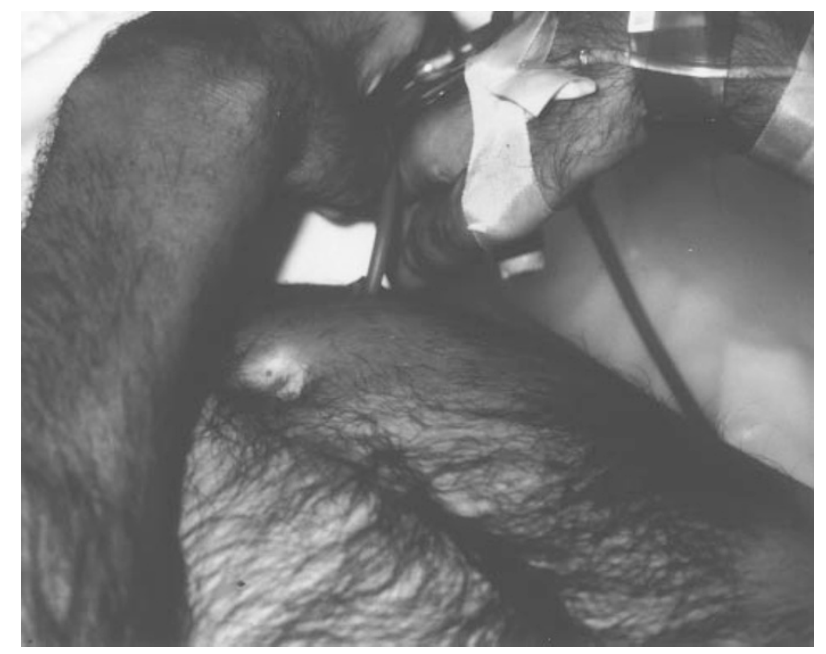

Figure 4 Picture of a spinal cord injury patient with limited manual dexterity utilizing the two-handed catheterization technique to catheterize his abdominal stoma

surgical technique that will augment their native bladder and thus increase their bladder capacity; and they also require a convenient, continent, easy-tocatheterize stoma to drain the augmented bladder. This is especially important to patients with cervical spinal cord injury, or debilitated patients with multiple sclerosis who are physically unable due to their condition to perform urethral self-catheterization. Patients whose manual dexterity is too limited to catheterize their native urethra adequately are often able to catheterize themselves via the abdominal stoma using a two-handed catheterization technique taught at our institution (Figure 4). Patients able to demonstrate their ability to perform the catheterization technique in the occupational therapy evaluation are potential candidates for this surgical technique.

Patients with progressive neurologic lesions and tetraplegia traditionally have not been considered for continent catheterizable bladder management options because of the expectation that they would be unable to perform self-catheterization. However, tetraplegic patients with bilateral pincer grip can be offered such a diversion after demonstrating their ability to perform two-handed catheterization (Figure 4). Diseases like multiple sclerosis, which have unpredictable clinical pregression, are even more problematic. Although we are concerned about the long-term hand function of these individuals, we have used the opinion of neurological consultants to aid our assessment of the appropriateness of continent diversion for each particular patient. A life expectancy (with appropriate function) of at least one to two years is a requirement for patients considered for the procedure.

Urethral closure, performed in seven patients, was undertaken only because these patients were incontinent at a low bladder pressure, had no urethra, or 
had a vesico-vaginal fistula. This procedure was not performed routinely because of the known high rate of fistula formation and spontaneous failure (Herschorn, 1993). Indeed two of the seven patients who had their urethras closed became incontinent, with one patient remaining incontinent via urethra and the other patient requiring three urethral closure procedures to finally establish urethral closure. In an additional four patients with low leak point pressures, the bladder neck and urethra were maintained but outlet resistance was increased either by injecting collagen to the bladder neck (one patient) or by performing a pubovaginal sling procedure (three patients). These procedures helped to establish urethral continence, allowed for an additional access route should difficulty with stomal catheterization arise, and served as a vent for elevated bladder pressures. ${ }^{8,9}$ All four of these patients remain continent via urethra and stoma, with the one patient who had collagen injection requiring repeat collagen injection to obtain urethral continence.

The continent vesicostomy segment created using this technique is necessary because either these patients failed urethral catheterization (due to limited dexterity or mobility) or have an incompetent urethral sphincter mechanism. The continence mechanism created by this technique has proved reliable and is easy to create surgically. The continence rate of $95 \%$ via urethra and stoma has remained constant throughout the study. With proper catheterization technique, the urinary infection rate has been extremely low and no metabolic abnormalities have been detected thus far using this technique. The intra-operative time is approximatley $4 \mathrm{~h}$ and the efferent limb has proved to be easily and reliably fashioned, with only two of 23 patients requiring re-operation.

Our results in 23 patients with an average follow-up of 26.9 months (range 3-67 months) and 95\% continence rate compare favorably with results previously reported by others. ${ }^{4}$ King et al reported on eight patients whose average follow-up was 33.9 months (range 9-64 months). Excellent continence and patient satisfaction were reported in $7 / 8$ patients with one patient preferring an indwelling catheter because of progressive neuromuscular disability. Our findings also compare favorably with the findings of Chao et al, who described seven patients who underwent a similar procedure with $100 \%$ immediate continence and a follow up of 23 months. ${ }^{10}$

To compare results using our technique with results using other techniques, one must critically examine what other techniques are available. One method that has been used employs a Kock ileal reservoir augmentation with a catheterizable nipple to the skin. ${ }^{11}$ Of the six patients described, four of the six are continent and one required re-operation for nipple dessusception (thus requiring reintussusception of the nipple with staples and Vicryl collar fixation). A HemiKock ileocystoplasty with a continent abdominal stoma has also been described..$^{8,12}$ Of the 18 patients described in Herschorn's paper, 17 of 18 patients remain continent (one lost to follow-up). Re-operations occurred in four of the 18 patients $(22 \%)$ to correct stomal incontinence, and two of these patients also developed bladder calculi. Results with our technique compare favorably with a comparable continence rate $(22 / 23)$ and lower re-operation rate (two of $23 ; 9 \%$ ). In addition, our technique avoids the well recognized nipple valve failure rate and risk of stone formation of at least $15 \%$ (and more often $33 \%$ ), even in experienced hands, ${ }^{13,14}$ by utilizing the easier-to-create Indiana continent efferent limb mechanism.

\section{Conclusions}

This modification of the Indiana continent urinary reservoir technique has proved itself to be a reliable and safe procedure, easy to perform and relatively free of significant complications. When utilized in the proper patient population, this technique offers good results and numerous benefits.

\section{References}

1 Simon J. Ectropia vesicae (absence of the anterior wall of the bladder and pubic abdominal parietes); operation for directing the orifices of the ureters into the rectum; temporary success; subsequent death; autopsy. Lancet 1852; 2: $568-570$.

2 Rowland RG and Kropp BP. Evolution of the Indiana continent urinary reservoir. J Urol 1994; 152: 2247-2251.

3 Rowland RG et al. Indiana continent urinary reservoir. J Urol 1987; 137: $1136-1139$.

4 Sarosdy MF. Continent urinary diversion using cutaneous ileocecocystoplasty. Urology 1992; 40: 102-106.

5 King DHC, Hlavinka TC, Sarosdy MF. Additional experience with continent urinary diversion using cutaneous ileocecocystoplasty. Urology 1996; 47: 471-475.

6 Bramble FJ. The treatment of adult eneusis and urge incontinence by enterocystoplasty. Br J Urol 1982; 54: 693-696.

7 Mitchell ME, Rink RC. Urinary diversion and undiversion. Urol Clin North Am 1985; 12: $111-112$.

8 Herschorn S, Thijssen AJ, Radomski SB. Experience with the hemi-Kock ileocystoplasty with a continent abdominal stoma. $J$ Urol 1993; 149: 998 - 1001

9 Keating MA, Rink RC, Adams MC. Appendicovesicostomy: a useful adjunct to continent reconstruction of the bladder. J Urol 1993; 149: 1091 - 1094.

10 Chao R, Mayo ME, Bejany DE, Bavendam T. Bladder neck closure with continent augmentation or suprapubic catheter in patients with neurogenic bladders. J Am Paraplegia Soc 1992; 16: $18-22$.

11 Bennett JK, Gray BG, Foote JE. Continent diversion and bladder augmentation in spinal cord-injured patients. Sem in Urol 1992; 10: $121-132$

12 Weinberg AC et al. The hemi-Kock augmentation ileocystoplasty: a low pressure anti-refluxing system. J Urol 1988; 140: $1380-1384$

13 deKernion JB et al. The Kock pouch as a urinary reservoir: pitfalls and perspectives. Am J Surg 1985; 150: 83 -89.

14 Waters WB, Vaughan DJ, Harris RG, Brady SM. The Kock pouch: initial experience and complications. J Urol 1987; 137: 1151 - 1153 . 